9. Wee LEI, Sim XYJ, Conceicao EP, et al. Containing COVID-19 outside the isolation ward: the impact of an infection control bundle on environmental contamination and transmission in a cohorted general ward. Am J Infect Control 2020;48:1056-1061.
10. Wee LE, Fan EMP, Heng R, et al. Construction of a container isolation ward: a rapidly scalable modular approach to expand isolation capacity during the coronavirus disease 2019 (COVID-19) pandemic. Infect Control Hosp Epidemiol 2020. doi: 10.1017/ice.2020.1222.

\title{
Frequent detection of severe acute respiratory syndrome coronavirus 2 (SARS-CoV-2) RNA on hands and skin of patients with coronavirus disease 2019 (COVID-19)
}

\author{
Sarah N. Redmond MD¹, Daniel F. Li MD²,2, Muhammed F. Haq MD², Lucas D. Jones BS ${ }^{3}$, Alexandria M. Nguyen MSHS \\ Margaret Tiktin DNP4 ${ }^{4}$, Jennifer L. Cadnum BS ${ }^{3}$, Maria E. Navas MD ${ }^{5}$, Jessica Bingham RN ${ }^{6}$, Brigid M. Wilson $\mathrm{PhD}^{7}$ and \\ Curtis J. Donskey MD ${ }^{1,7}$ \\ ${ }^{1}$ Case Western Reserve University School of Medicine, Cleveland, Ohio, ${ }^{2}$ Research Service, Louis Stokes Cleveland VA Medical Center, Cleveland, Ohio, \\ ${ }^{3}$ Department of Molecular Biology and Microbiology, Case Western Reserve University School of Medicine, Cleveland, Ohio, ${ }^{4}$ Clinical Research Center, Louis \\ Stokes Cleveland VA Medical Center, Cleveland, Ohio, ${ }^{5}$ Pathology and Laboratory Medicine Services, Louis Stokes Cleveland VA Medical Center, Cleveland, Ohio, \\ ${ }^{6}$ Nursing Service, Louis Stokes Cleveland VA Medical Center, Cleveland, Ohio and ${ }^{7}$ Geriatric Research, Education, and Clinical Center, Louis Stokes Cleveland VA \\ Medical Center, Cleveland, Ohio
}

To the Editor-Hand hygiene is a core measure recommended for prevention of severe acute respiratory syndrome coronavirus 2 (SARS-CoV-2) transmission. ${ }^{1,2}$ Hand hygiene is needed to eliminate SARS-CoV-2 acquired on hands during contact with infected patients or contaminated surfaces and fomites, including personal protective equipment. ${ }^{1-3}$ In an in vitro model, SARS-CoV-2 inoculated onto human skin survived for 9 hours after inoculation versus 2 hours for influenza A. ${ }^{4}$ SARS-CoV-2 was rapidly inactivated on skin by $80 \%$ ethanol. $^{4}$

Hand hygiene may also be beneficial in reducing the burden of SARS-CoV-2 on the hands of individuals with coronavirus disease 2019 (COVID-19), and bathing may reduce SARS-CoV-2 on other skin sites. However, the burden of SARS-CoV-2 on hands and other skin sites of COVID-19 patients is unknown. Here, we tested the hypothesis that SARS-CoV-2 RNA is frequently present on the hands and the chest/abdomen of patients with COVID-19, particularly those with a high viral burden in samples collected from the anterior nares.

The study protocol was approved by the Cleveland VA Medical Center's Institutional Review Board. Between November 1, 2020, and April 26, 2021, a convenience sample of patients with symptomatic COVID-19 was enrolled. Patients were excluded if they had dementia or delirium, were admitted to the intensive care unit, and if $>3$ days had passed since their COVID-19 diagnosis.

Premoistened CLASSIQSwabs with universal transport medium (Copan Diagnostics, Murrieta, CA) were used to collect samples from the anterior nares, oropharynx, chest/abdomen $(10 \times 10-\mathrm{cm}$ surface area), and the entire surface area of both hands. Swabs were also used to sample environmental surfaces in patient rooms, including $5 \times 20-\mathrm{cm}$ areas of the bed rail and bedside table; outpatients sampled a frequently used chair and tabletop. A medical record

\footnotetext{
Author for correspondence: Curtis J. Donskey, E-mail: Curtis. Donskey@va.gov Cite this article: Redmond SN, et al. (2022). Frequent detection of severe acute respiratory syndrome coronavirus 2 (SARS-CoV-2) RNA on hands and skin of patients with coronavirus disease 2019 (COVID-19). Infection Control \& Hospital Epidemiology, 43: 1976-1977, https://doi.org/10.1017/ice.2021.403
}

review was conducted to obtain information on age, sex, medical conditions, and COVID-19 treatments.

A detailed description of the methods for RNA extraction and reverse transcriptase quantitative polymerase chain reaction (RT-qPCR) is included as Supplementary Material (online). The Fisher exact test was used to compare the proportions of COVID-19 patients with positive chest/abdomen or hand SARS-CoV-2 RNA for those with anterior nares cycle threshold $\left(C_{t}\right)$ values of $\leq 30$ or $>30$. The cycle threshold cutoff of 30 was chosen based on evidence that individuals with higher nasopharyngeal swab $C_{t}$ values are less likely to have a positive cultures or to transmit SARS-CoV-2 to contacts. ${ }^{5}$ Analyses were performed using $\mathrm{R}$ version 3.5.1 statistical software ( $\mathrm{R}$ Foundation for Statistical Computing, Vienna, Austria).

For the 16 study participants, the mean age was 60.8 years; $13(81.3 \%)$ were male; $9(56.3 \%)$ were receiving dexamethasone and supplemental oxygen at the time of enrollment; and 2 (12.5\%) were outpatients. The anterior nares RT-qPCR result was positive for SARS-CoV-2 in all participants with an average $C_{t}$ value of 29.2 (range, 18.2-40). Figure 1.A shows the percentage of other sites with positive RT-qPCR results for SARS-CoV-2 RNA, stratified by anterior nares $C_{t}$ value $\leq 30$ or $>30$. Of the 16 participants, the oropharynx, chest/abdomen, hand, and environmental samples were positive for SARS-CoV-2 RNA in 15 (93.8\%), 7 (43.8\%), 7 (43.8\%), and $3(18.8 \%)$ patients, respectively. All 7 patients with SARS-CoV-2 RNA on hands also had positive chest/abdomen samples. The percentage of chest/abdomen and hand samples that were positive was significantly higher in patients with anterior nares $\mathrm{C}_{\mathrm{t}}$ values of $\leq 30$ versus $>30$ : $6(75 \%)$ of 8 versus $1(12.5 \%)$ of $8(P=.04)$.

Figure $1 \mathrm{~B}$ shows a boxplot of the $\mathrm{C}_{t}$ values for SARS-CoV-2 RNA from all sites. Of the 7 patients with positive chest/abdomen RT-qPCR results for SARS-CoV-2, 1 patient (14.2\%) had a chest/ abdomen $C_{t}$ value $<30$ and 3 patients $(42.9 \%)$ had $C_{t}$ values between 30 and 35 . Of the 7 patients with positive hand RT-qPCR results for SARS-CoV-2, 3 patients (42.9\%) had hand $\mathrm{C}_{\mathrm{t}}$ values between 30 and 35 . Only $1(16.7 \%)$ of 6 positive 
(A)

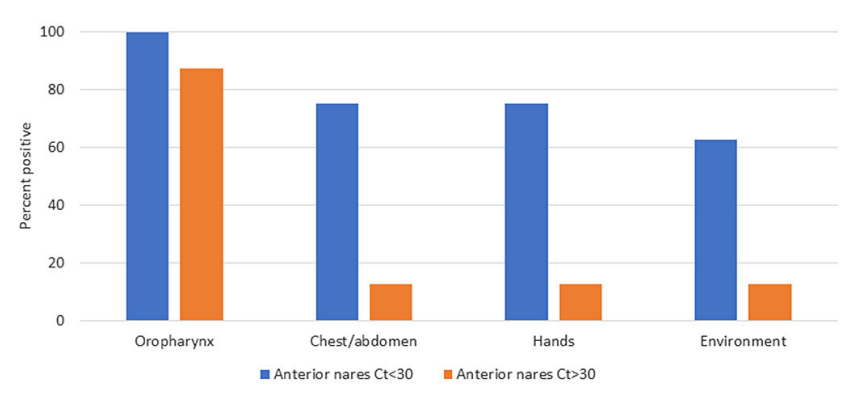

(B)

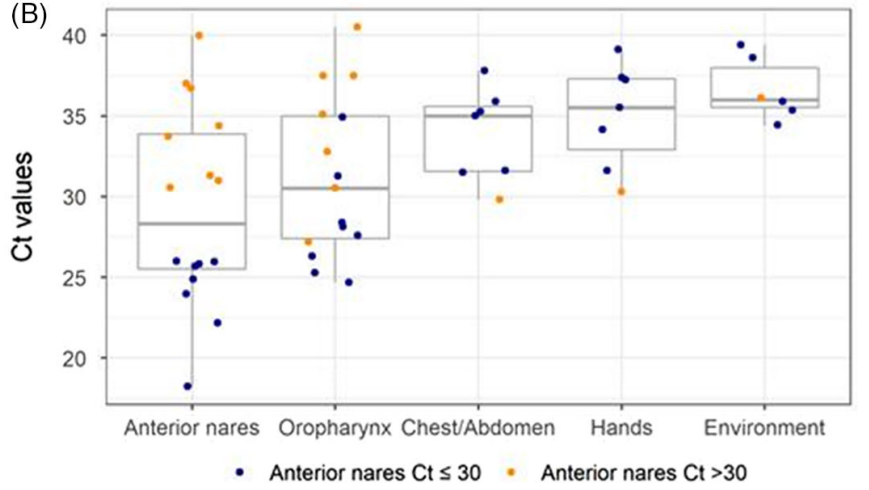

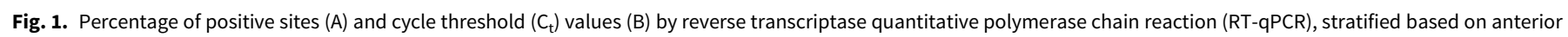
nares $C_{t}$ values of $\leq 30$ and $>30$.

environmental samples had RT-qPCR for SARS-CoV-2 with a $\mathrm{C}_{t}$ value $<35$.

Nearly half of COVID-19 patients had detectable levels of SARSCoV-2 RNA on their hands and chest/abdomen. Contamination was significantly more common in patients with an increased nasal burden of SARS-CoV-2 based on an anterior nares $\mathrm{C}_{\mathrm{t}}$ value of $<30$ ( $75 \%$ vs $12.5 \%)$. The burden of SARS-CoV-2 detected on the hands and chest/abdomen was relatively low, but approximately half had $\mathrm{C}_{\mathrm{t}}$ values of $<35$ and 1 chest/abdomen sample had a $C_{t}$ value of $<30$.

Our findings suggest that the hands and other skin sites of patients with COVID-19 could be an important source of transmission. Frequent hand hygiene should be emphasized for patients with suspected or confirmed COVID-19. Because presymptomatic patients contribute substantially to transmission of SARS-CoV-2, ${ }^{6,7}$ hand hygiene is also essential in the absence of symptoms. Educational interventions can increase hand hygiene in hospitalized patients and long-term care facility residents. ${ }^{8}$ Because handshake and fist bump greetings efficiently transfer viruses, ${ }^{9}$ noncontact greetings should be encouraged. Finally, frequent bathing may be beneficial in reducing the burden of virus on skin. Chlorhexidine bathing is commonly used in healthcare facilities and has been shown to be effective against SARS-CoV-2. ${ }^{10}$

Our study has several limitations. The swabs used for sampling the hands and chest/abdomen are designed for collection of nasopharyngeal specimens and were likely suboptimal for recovery of virus from skin. It is plausible that our results underestimate the burden of SARS-CoV-2 on skin. We did not perform viral cultures and cannot exclude the possibility that the SARS-CoV-2 RNA detected on skin represents nonviable virus. However, SARSCoV-2 has been shown to survive for hours on skin. ${ }^{4}$

Supplementary material. To view supplementary material for this article, please visit https://doi.org/10.1017/ice.2021.403

Acknowledgments. We thank the patients for their participation in the study.
Financial support. This work was supported by a Merit Review grant (no. CX001848) from the Department of Veterans' Affairs to C.J.D.

Conflicts of interest. C.J.D has received research grants from Clorox, Pfizer, and PDI. All other authors report no conflicts of interest relevant to this article.

\section{References}

1. How to protect yourself and others. Centers for Disease Control and Prevention website. https://www.cdc.gov/coronavirus/2019-ncov/preventgetting-sick/prevention.html. Accessed July 31, 2021.

2. Interim infection prevention and control recommendations for patients with suspected or confirmed coronavirus disease 2019 (COVID19) in healthcare settings. Centers for Disease Control and Prevention website. https://www.cdc.gov/coronavirus/2019-ncov/hcp/infection-controlrecommendations.html. Accessed July 31, 2021.

3. Li DF, Alhmidi H, Scott JG, et al. A simulation study to evaluate contamination during reuse of $\mathrm{N} 95$ respirators and effectiveness of interventions to reduce contamination. Infect Control Hosp Epidemiol 2021. doi: 10.1017/ice. 2021.218.

4. Hirose R, Ikegaya H, Naito Y, et al. Survival of SARS-CoV-2 and influenza virus on the human skin: Importance of hand hygiene in COVID-19. Clin Infect Dis 2020. doi: 10.1093/cid/ciaa1517.

5. Lee LYW, Rozmanowski S, Pang M, et al. SARS-CoV-2 infectivity by viral load, $S$ gene variants and demographic factors and the utility of lateral flow devices to prevent transmission. Clin Infect Dis 2021. doi: 10.1093/cid/ ciab421.

6. Johansson MA, Quandelacy TM, Kada S, et al. SARS-CoV-2 transmission from people without COVID-19 symptoms. JAMA Netw Open 2021;4: e2035057.

7. Jones LD, Chan ER, Zabarsky TF, et al. Transmission of SARS-CoV-2 on a patient transport van. Clin Infect Dis 2021. doi: 10.1093/cid/ciab347.

8. Sunkesula VCK, Kundrapu S, Knighton S, Cadnum JL, Donskey CJ. A randomized trial to determine the impact of an educational patient hand-hygiene intervention on contamination of hospitalized patient's hands with healthcare-associated pathogens. Infect Control Hosp Epidemiol 2017;38:595-597.

9. Jones LD, Ha W, Pinto-Herrera N, et al. Transfer of bacteriophage MS2 by handshake versus fist bump. Am J Infect Control 2020;48:727-729.

10. Hirose R, Bandou R, Ikegaya $\mathrm{H}$, et al. Disinfectant effectiveness against SARS-CoV-2 and influenza viruses present on human skin: model-based evaluation. Clin Microbiol Infect 2021. doi: 10.1016/j.cmi.2021.04.009. 